\title{
Behavior of Gold-Doped Silicon Substrate under Small- and Large-RF Signal
}

\author{
Massinissa Nabet ${ }^{1 a}$, Martin Rack ${ }^{1}$, C. H. (Kees) de Groot ${ }^{2}$ and Jean-Pierre Raskin ${ }^{1 b}$ \\ ${ }^{1}$ ICTEAM, Université catholique de Louvain, 1348 Louvain-la-Neuve, Belgium \\ ${ }^{2}$ School of Electronics and Computer Science, University of Southampton, Southampton SO17 1BJ, UK \\ ${ }^{a}$ massinissa.nabet@uclouvain.be, ${ }^{b}$ jean-pierre.raskin@uclouvain.be
}

\begin{abstract}
In this paper, small- and large-signal performances of passive devices integrated on high-resistivity, trap-rich and gold-doped silicon wafers are presented and compared through measurements and simulations. The gold-doped silicon substrate was produced starting from standard silicon having a nominal resistivity of $56 \Omega . \mathrm{cm}$. We show that the gold-doped substrate presents high effective resistivity and low losses suitable for RF applications, this has been done by measuring coplanar waveguides, crosstalk, inductors and band pass filter where we saw a similar performances under small signal-measurements. Largesignal measurements of gold-doped substrates show $60 \mathrm{dBm}$ lower harmonic distortion than high-resistivity substrates, and 10 dB lower than trap-rich substrate. A large DC bias dependence on the harmonic distortion induced by the gold-doped substrate is observed. This unexpected behavior is explained using the Fermi level localization in the silicon bandgap for the different DC bias conditions.
\end{abstract}

Keywords-Gold-doped silicon substrate; Silicon-on-Insulator (SOI) technology; effective resistivity; substrate modeling; RF substrate; harmonic distortion (HD); trap-rich (TR) highresistivity (HR) silicon.

\section{INTRODUCTION}

The microelectronic market is demanding more and more innovation and new capabilities in mobile devices, this is why the communications industry is looking for reliable fabrication processes and substrates that can meet all RF requirements [1]. Choosing the right substrate technology is an important step in order to cointegrate the digital devices, analog and RF frontend modules on the same substrate. Silicon technology has been considered as the promising solution for RF applications and communication systems due to its low cost, maturity and its continuous improvement [2]. The design and the fabrication of RF circuits (PA, LNA, switch...etc) are challenged by the substrate upon which the devices are built, because it is the main source for electromagnetic coupling, RF losses and nonlinearities. Many silicon substrates have been developed in the past starting with standard silicon substrate (nominal resistivity around $20 \Omega-\mathrm{cm}$ ), however the digital noise coming from digital devices will propagate through the bulk and affect the performance of analog and RF devices built on this substrate, thereby reducing the ability of co-integration of the RF devices on the same substrate (SiPs and SoCs). New integration levels were reached thanks to the optimization of the silicon substrate [3]. Silicon substrates with high effective resistivity [4] (higher than $3 \mathrm{k} \Omega . \mathrm{cm}[5])$ are the most appropriate for RF applications due to the high quality passives, less RF loss, decreased crosstalk and reduced signal distortion.

High-resistivity silicon-on-insulator (HR-SOI) substrates were introduced to be a good candidate for RF applications thanks to its high isolation, low substrate loss and low cost [6]-[8]. Although the HR substrate is significantly low doped (nominal resistivity as high as $3 \mathrm{k} \Omega . \mathrm{cm}$ ) compared to standard substrate, its RF performances are barely improved [9], [10]. The main problem of HR substrate is the Parasitic Surface Conduction (PSC) originating from the fixed charges in the insulating layer which attract free carriers from the substrate to the $\mathrm{Si}-\mathrm{SiO}_{2}$ interface and create a highly conductive layer creating inhomogeneity in the substrate thereby reducing its effective resistivity [4]. The trap-rich substrate resolves the problem of PSC by introducing a thin layer of polysilicon (between $300 \mathrm{~nm}$ and $2 \mu \mathrm{m}$ ) between the Si and the insulating layer. Polysilicon introduces a large amount of defects that trap the free carriers at the interface, theses traps pin the Fermi level near the silicon midgap, therefore the substrate recover its high effective resistivity.

A new concept called deep level doping compensation was introduced in order to increase the resistivity of SOI substrate, consequently reducing the PSC effects by introducing a high density of traps in the entire silicon substrate thickness [11]. The effects of background free carriers are compensated by implanted dopant atoms, thus reducing the carrier concentration, consequently increasing the resistivity of the silicon substrate [12]. The material choosen for doping is gold, because it can be used for both n-type and p-type silicon in contrast to other dopants that only introduce either donors or acceptors. Gold also introduces equal concentrations of donors and acceptors at energy levels near the midgap [13]. Doping with gold does not require exact control of the quantity of dopants to achieve the resistivity needed, and also the gold is among most studied materials in the litterature [14]-[16]. Deep level compensation technique has showed its capabilities to improve the RF performances of the substrate by reducing attenuation from $0.8 \mathrm{~dB} / \mathrm{mm}$ to 0.3 $\mathrm{dB} / \mathrm{mm}$ [17], for CPW fabricated on gold doped silicon and standard silicon respectively. Resistivities arround $100 \mathrm{k} \Omega . \mathrm{cm}$ have been reached for gold doped silicon substrate [18]. It was also shown that this technique can supress the PSC effects and eliminate the bias dependence [11], however the non-linearities of the gold-doped silicon substrate and its performances under large RF signal have not been reported in the literature yet. 
In this paper, we present the behavior of the Au-Si substrate under small- and large-RF signal. In section II we will describe the experimental results under small RF signal and the characteristics of the CPW line including the behavior of the effective resistivity, crosstalk measurements, inductor quality factor, band pass filter, then we show the harmonic distortion measurements. In section III, we will explain the strange behavior of the non-linearities in gold doped silicon substrate using Fermi level extraction and the impedance variations, the location and the effect of trap densities will be shown as well.

\section{EXPERIMENT}

\section{A. Substrates Description}
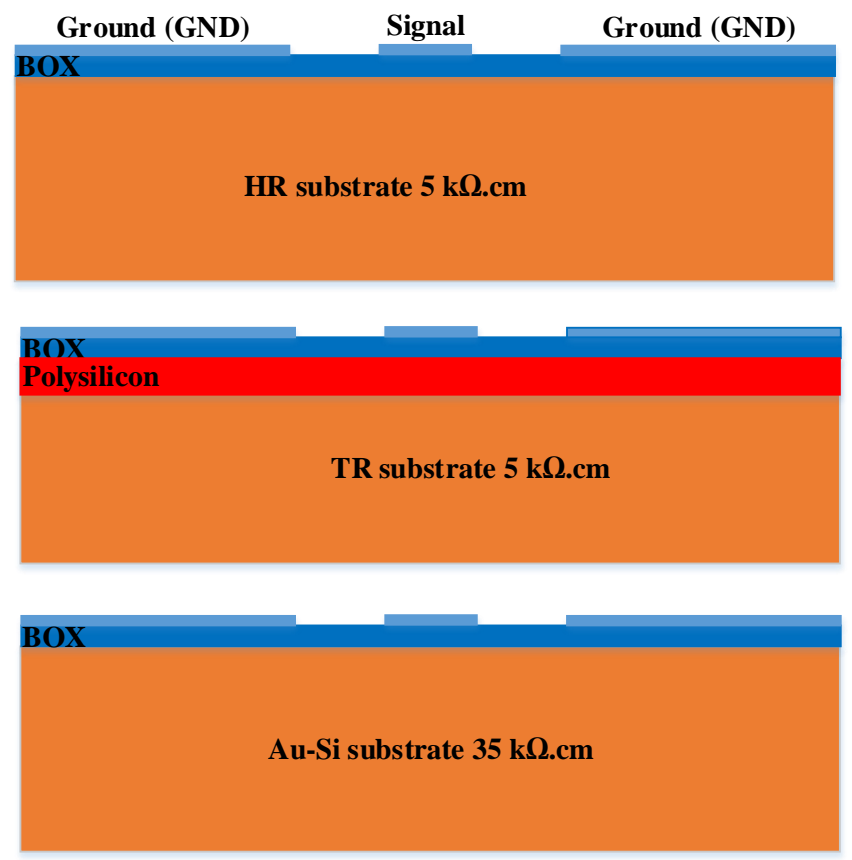

Fig. 1: The three Si-based substrates fabricated

Coplanar waveguides (CPW) were fabricated for RF characterization on three types of high-resistivity silicon substrates (Fig. 1) with $700 \mu \mathrm{m}$ thickness: (i) n-type Si substrate with 5 $\mathrm{k} \Omega . \mathrm{cm}$ nominal resistivity (HR), (ii) high resistivity $\mathrm{Si}$ with a 1 $\mu \mathrm{m}$ thin layer of trap rich polysilicon (TR), and (iii) Au-doped $\mathrm{Si}$ substrate $(\mathrm{Au}-\mathrm{Si})$. For this last substrate we started with a standard n-type silicon wafer with a nominal resistivity of 56 $\Omega . \mathrm{cm}$. Gold atoms were then implanted at the backside of the wafer with a dose of $4 \times 10^{13} \mathrm{~cm}^{-2}$ at an energy of $100 \mathrm{keV}$. After implantation, the gold atoms are not yet activated and do not contribute to the resistivity increase. This is why the wafer was subsequently annealed at $950{ }^{\circ} \mathrm{C}$. After that we obtained a substrate with a nominal resistivity of around $35 \mathrm{k} \Omega-\mathrm{cm}$. All substrates have an insulating layer with thickness of $\mathrm{t}_{\mathrm{ox}}=$ $200 \mathrm{~nm}$ that was deposited using low-pressure chemical vapor deposition (LPCVD). The $50 \Omega \mathrm{CPW}$ line was defined as: $\mathrm{W}_{\mathrm{c}}=26 \mu \mathrm{m}$ (central signal conductor width), $\mathrm{S}=12 \mu \mathrm{m}$ (signalto-ground line spacing) and $\mathrm{W}_{\mathrm{g}}=208 \mu \mathrm{m}$ (ground conductor width). Finite element simulations were carried out using Atlas software which simulates accurately the physical parameters such as fixed oxide charges, interface and volume traps by resolving Poisson's and semiconductor equations in the Sibased substrates. The density of positive fixed oxide charges $\mathrm{Q}_{\mathrm{ox}}$ was set at $10^{11} \mathrm{~cm}^{-2}$ for all substrates. The spatial $\mathrm{Au}$ distribution was defined based on experimental SIMS data presented in [17]. The trap-rich substrate was simulated using tail distribution for donor and acceptor traps to model the polysilicon layer according to the model given in [20], and using characteristic decay energy of $33 \mathrm{meV}$ and edge band state density of $10^{22} \mathrm{~cm}^{-3} \mathrm{eV}^{-1}$.

\section{B. Simulated local resistivity profile}

In Fig. 2 are depicted the local resistivity profiles below the insulating layer simulated for the three different substrates (HR, TR and Au-Si) using the finite element semiconductor simulation tool Atlas [19] from Silvaco.

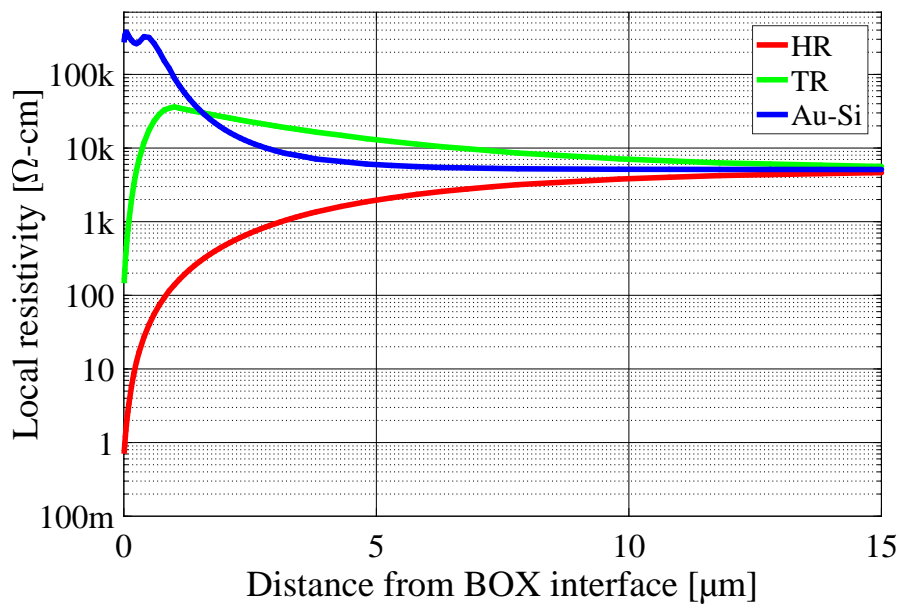

Fig. 2: Simulated resistivity profiles in the silicon volume below the insulating layer for the three Si-based substrates.

It appears clearly that HR substrate suffers from the PSC effect due to the low resistivity layer near the $\mathrm{Si}-\mathrm{SiO}_{2}$ interface and shows a local value around $700 \mathrm{~m} \Omega . \mathrm{cm}$. The trap-rich which is a solution to the PSC problem shows better performances with a local resistivity near the $\mathrm{Si}_{-} \mathrm{SiO}_{2}$ interface higher than the later, this is due to the traps generated by the polysilicon layer. The Au-Si substrate does not suffer from the PSC effects and shows values higher than $200 \mathrm{k} \Omega . \mathrm{cm}$ near the $\mathrm{Si}-\mathrm{SiO}_{2}$ interface. This is due to the gold atoms that create acceptor and donor levels in the silicon band-gap and close to the midgap, this latter compensates the free carriers that are in the substrate.

\section{Effective Resistivity as a Function of Frequency}

The small-signal measurements of the CPW lines were performed using an Agilent 2-port performance network analyzer (PNA)-X vector network analyzer and a pair of ground-signal ground (GSG) $|\mathrm{Z}|$ probes from Cascade Microtech with $150 \mu \mathrm{m}$ pitch. The effective resistivity was extracted from S-parameters measurements. We used 
short-open-thru-load (SOLT) calibration method in order to calibrate the system at the probe tips. The deembeding proposed by Gillon [21] was used in order to remove the pad parasitics by measuring the open and the thru on-wafer after measuring the $\mathrm{CPW}$ line.

The small-signal measurements and simulations of the CPW line are depicted in Fig. 3. We can see that the simulations agree well with the measurements.

The HR substrate suffers from PSC: the effective resistivity is therefore low as expected, and shows a value of around 40 $\Omega . \mathrm{cm}$ at $4 \mathrm{GHz}$. This low value translates to an increase in the CPW line's RF losses. The TR substrate effectively mittigates the PSC effect and its effective resistivity is reletively high around $10 \mathrm{k} \Omega-\mathrm{cm}$ at $4 \mathrm{GHz}$. The Au-Si substrate shows better performances than TR, with an effective resistivity reaching a value of more than $15 \mathrm{k} \Omega . \mathrm{cm}$ at $4 \mathrm{GHz}$. The gold atoms in substitutional sites create traps in the bandgap of the silicon, deep donors and deep acceptors at $\mathrm{E}_{\mathrm{C}}-0.78 \mathrm{eV}(0.78 \mathrm{eV}$ below conduction band) and $\mathrm{E}_{\mathrm{V}}+0.58 \mathrm{eV}(0.58 \mathrm{eV}$ above valence band), respectively [22]. The traps created by gold atoms capture free charges in the silicon bulk, the Fermi level at the $\mathrm{Si}-\mathrm{SiO}_{2}$ interface is located near the mid-gap, thereby increasing the overall resistivity.

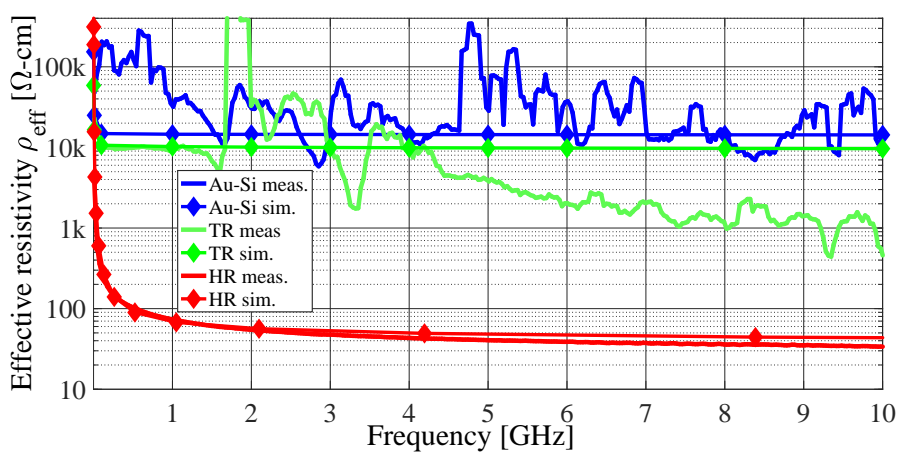

Fig. 3: Measurement and simulation results of the effective resistivity extracted from $\mathrm{CPW}$ lines designed on the three different substrates at $0 \mathrm{~V} \mathrm{DC}$ bias.

\section{Effective Resistivity Bias Dependance}

To better understand of the behavior of the substrates, we extracted the effective resistivity as a function of the DC bias points of the CPW line which is depicted in Fig. 4.

The effective resistivity of the HR substrate is bias dependent, as we can see it is around $40 \Omega$-cm for positive bias points and around $130 \Omega-\mathrm{cm}$ for negative bias points. When applying a positive DC bias we create more electrons below the signal electrode, so that enhance the PSC effect, therefore we register low effective resistivity. When we apply a negative DC bias, we are creating holes below the signal electrode, this leads to the creation of a depletion region between the signal and ground electrodes. The electric field will face a high resistive region between the signal and ground electrodes, so the effective resistivity is relatively high compared to when we apply a positive DC bias.

For TR and Au-Si substrates, the effective resistivity is bias independent and is around $10 \mathrm{k} \Omega-\mathrm{cm}$ and $35 \mathrm{k} \Omega-\mathrm{cm}$, respectively. The bias independency is due to the traps created by the polysilicon and gold atoms, so when we generate electrons (and holes) in the substrate by applying a positive (and negative) DC bias, the traps will capture them and reduce their lifetime.

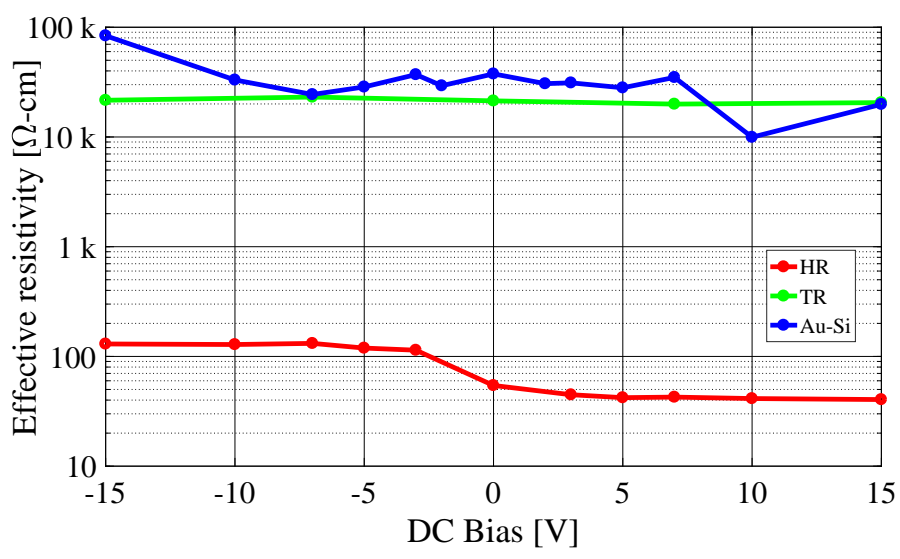

Fig. 4: Extraction of the effective resistivity as a function of DC bias for the Si-based substrates at $2 \mathrm{GHz}$

\section{E. Substrate Crosstalk Levels}

Since the integration of RF and millimeter-wave devices on the same substrate became crucial, it is of first importance to reduce the coupling through the substrate. The RF coupling is caused by the neighboring devices especially RF switches and digital components. The assessment of the crosstalk level was performed by the passive structure shown in the inset of Fig. 5 that was carried out and measured on the three siliconbased substrates described above. The crosstalk pads have the specifications: length $\mathrm{L}$ of $150 \mu \mathrm{m}$, the spacing between the two pads is $50 \mu \mathrm{m}$ and the width $\mathrm{W}$ of $50 \mu \mathrm{m}$.

The HR substrate shows strong coupling curve compared to TR and Au-Si and it shows a flattening tendency between 100 $\mathrm{MHz}$ and $2 \mathrm{GHz}$ that represents the resistive behavior of the substrate. This value around $-40 \mathrm{~dB}$ is relatively high due to the low effective resistivity of the substrate created by the PSC effect that facilitates the coupling in the first $100 \mathrm{~nm}$ below the insulating layer. The slope of $20 \mathrm{~dB} / \mathrm{dec}$ below $100 \mathrm{MHz}$ is due to the insulating layer capacitance. However, the slope above $20 \mathrm{GHz}$ is due to the substrate capacitance, and since all the studied substrates are silicon-based (they are showing an effective permittivity of 9), we notice that the three curves join each other.

The TR and Au-Si show similar coupling because they are highly resistive due the traps contained in the two substrates. We can notice that theses curves do not show a flattening behavior, this is because it is so small that it does not appear ( below a few MHz). The curves of these two substrates shows only the capacitive behavior of the substrate. 


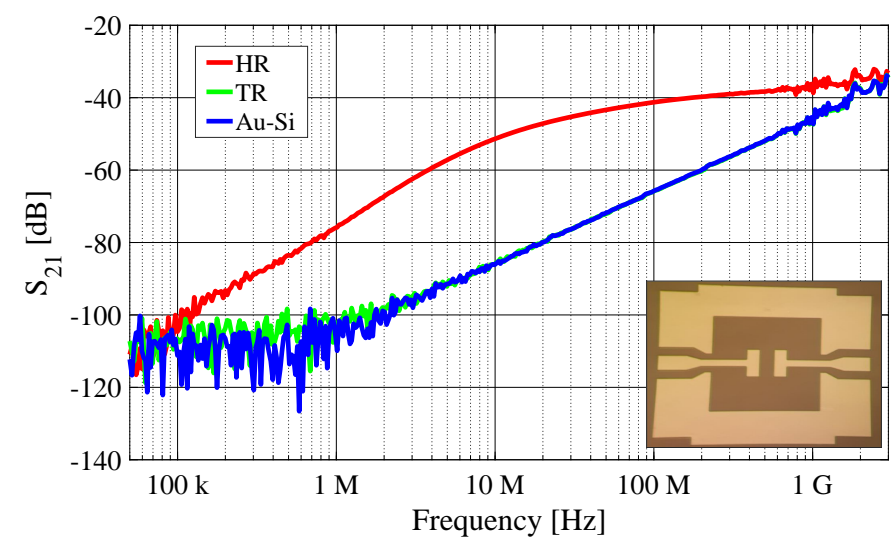

Fig. 5: Comparison of the measured crosstalk levels on the various Si-based substrates under consideration.

\section{F. Quality Factor Measurements}

In order to investigate the behavior of the substrate on the performances of inductors, we designed an inductor having the following characteristics: the spacing between the lines $S=10$ $\mu \mathrm{m}$, the width of the lines $\mathrm{W}=40 \mu \mathrm{m}$, the inner radius $\mathrm{Ri}=100$ $\mu \mathrm{m}$ and the number of turns $\mathrm{N}=3.5$. After the measurement of the $\mathrm{S}$ parameters we extracted the values of $\mathrm{R}$ and $\mathrm{L}$ then the quality factor was computed as: :

$$
\mathrm{Q}=\frac{2 \pi * \mathrm{f} * \mathrm{~L}}{\mathrm{R}}
$$

Where $\mathrm{R}$ and $\mathrm{L}$ are the internal resistance and the reactance of the inductor, respectively. The value of the inductor $\mathrm{L}$ is found $3.6 \mathrm{nH}$ (The value at low frequencies between 0.4 and $4 \mathrm{GHz}$ ) and was plotted in Fig. 6. The HR substrate show a maximum Qf around 5 at $2 \mathrm{GHz}$, that is relatively low due to the PSC effect. In contrast, the TR substrate shows a value around 16 at $6 \mathrm{GHz}$ and is close to that of $\mathrm{Au}-\mathrm{Si}$ (around 20 at $6 \mathrm{GHz}$ ), this is because of the high resistivity offered by the two subtrates.

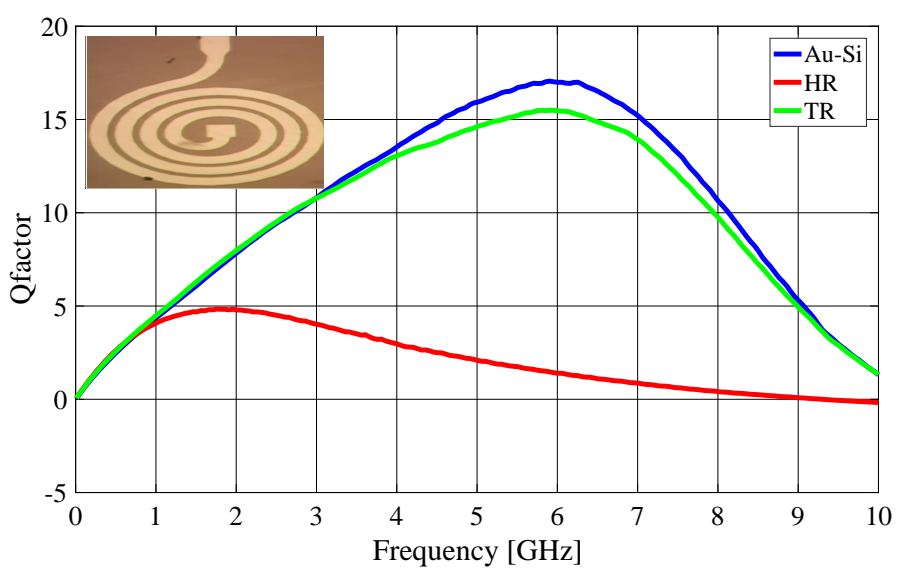

Fig. 6: The value of the quality factor as a function of frequency for inductor integrated on the HR, TR and $\mathrm{Au}-\mathrm{Si}$ substrates.

\section{G. Coupled Line Bandpass Filter Measurements}

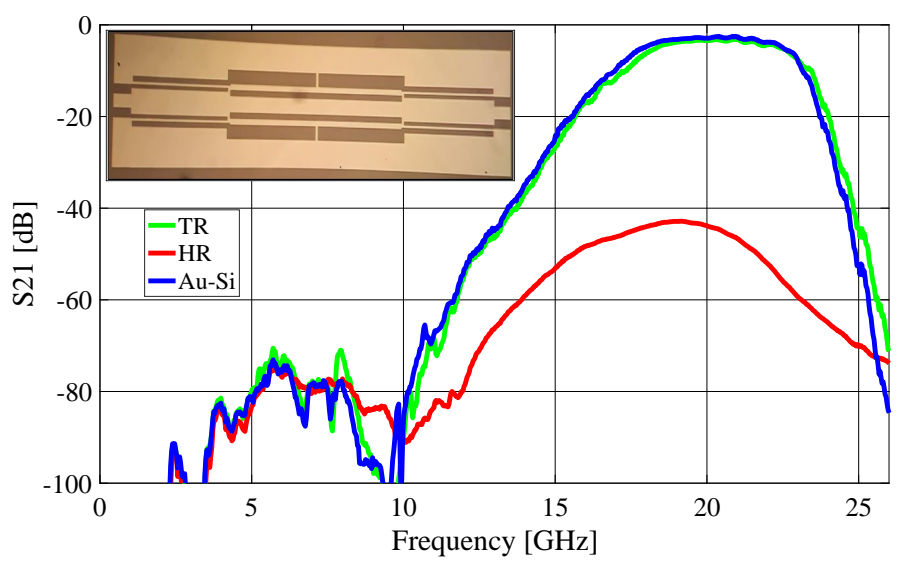

(a) Transmission coefficient as a function of frequency

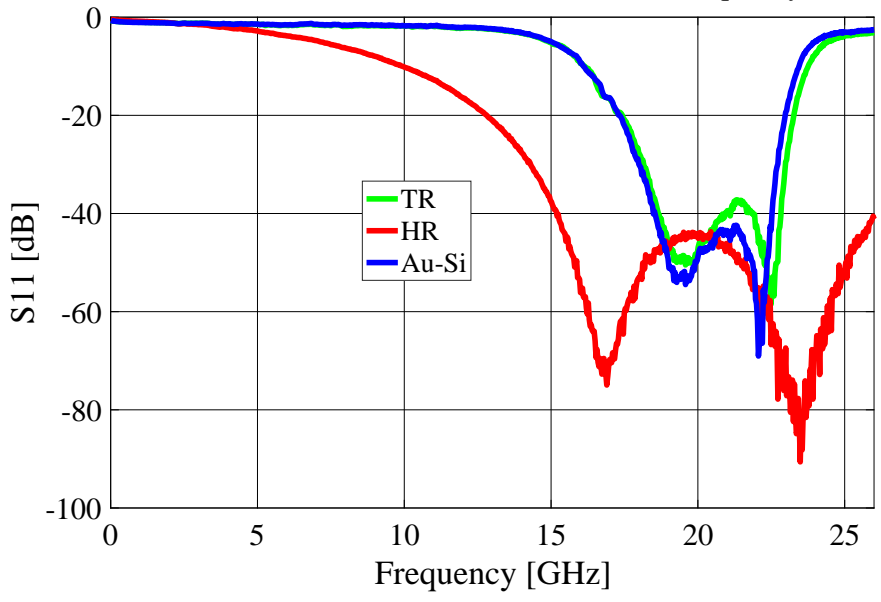

(b) Reflection coefficient as a function of frequency

Fig. 7: Evolution of the $S_{11}$ (a) and $S_{21}$ (b) values of the bandpass filter with the frequency for the three studied substrates

We also characterizing the substrates by designing a 18-22 $\mathrm{GHz}$ coupled line band pass filter that was implemented on the three silicon-based substrates. Fig. 7 shows the behavior of the designed filter during reception (a) and transmission (b), the picture of the filter is in the inset of Fig. 7a. It is shown in Fig. 7a that the filter implemented in HR substrate is bad in term of insertion loss (S21) and presents a maximum value of $-40 \mathrm{~dB}$, this is due to the the substrate loss caused by the PSC effect. However, TR and Au-Si substrates offer a bandwidth of $5 \mathrm{GHz}(17$ to $22 \mathrm{GHz}$ ) and are so close to the design and presents more than $40 \mathrm{~dB}$ gain better than HR substrates because of the traps created by both poly-silicon layer and gold atoms. Fig. $7 \mathrm{~b}$ shows the reflection coefficient of the studied substrates, where we confirm the good performances of TR and Au-Si substrates over the HR.

\section{H. Large-Signal Measurements}

In the case of passive devices fabricated on Si-based substrates it was shown that the harmonic distortion comes from the substrate itself and not from the metallic lines [23], 
connectors or contact surfaces. The distribution of free carriers in the substrate is modulated by the electric field applied to the CPW line.

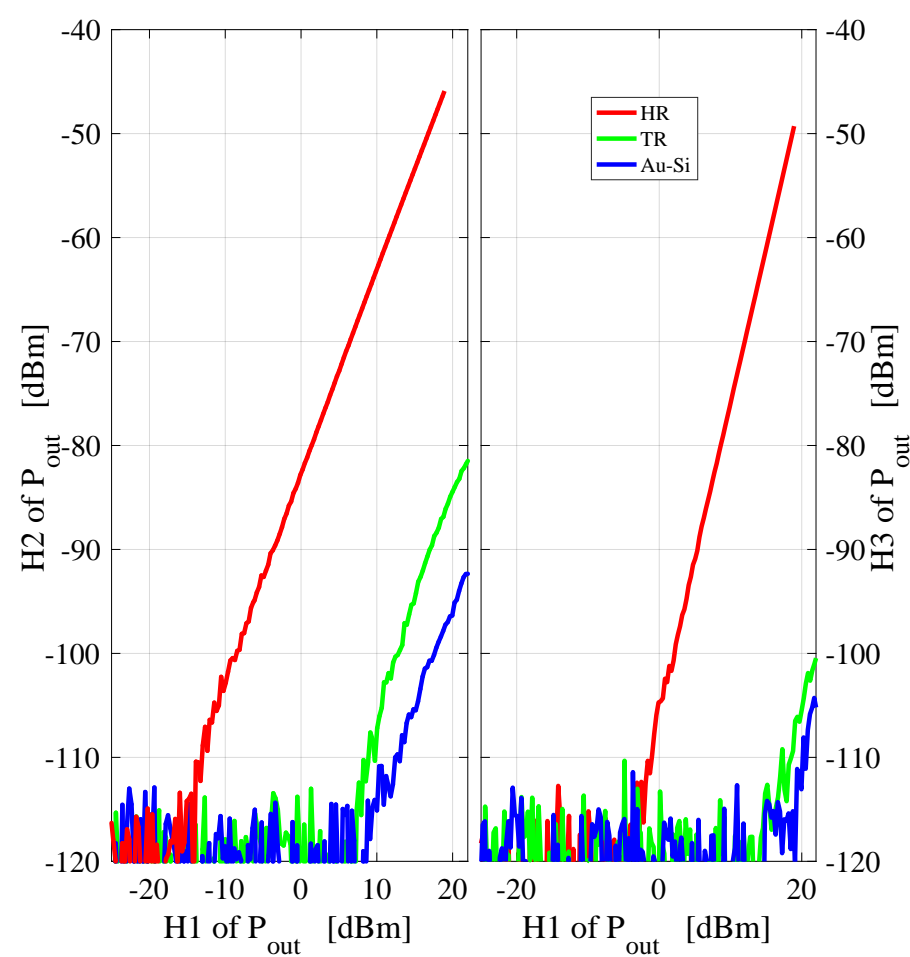

Fig. 8: Measured H2 (left) and $\mathrm{H} 3$ (right) for CPW line built on $\mathrm{Au}-\mathrm{Si}, \mathrm{HR}$ and substrates versus fundamental $\mathrm{H} 1$ output power at $900 \mathrm{MHz}$ and at $0 \mathrm{~V} \mathrm{DC}$ bias.

For the large-signal measurements, a single tone with a fundamental frequency of $900 \mathrm{MHz}$ is injected into one port of the CPW line by varying the power from -25 to $25 \mathrm{dBm}$. The harmonics $\mathrm{H} 2$ and $\mathrm{H} 3$ measured at the output of the CPW line are plotted versus fundamental output power $\mathrm{H} 1$ in Fig. 8 for the Si-based substrates described above at $0 \mathrm{~V} \mathrm{DC}$ bias. It is shown that the HR substrate presents high harmonic distortion and shows a value of the second harmonic of -53.37 $\mathrm{dBm}$ at an input power of $15 \mathrm{dBm}$ due to its low effective resistivity (around $40 \Omega \mathrm{cm}$ ). The TR substrate shows better linearity compared to $\mathrm{HR}(\mathrm{H} 2=-95.6 \mathrm{dBm}$ at an input power of $15 \mathrm{dBm}$ ) due to its higher resistivity (around $10 \mathrm{k} \Omega \mathrm{cm}$ ). The Au-Si shows a second harmonic of $-111 \mathrm{dBm}$ at an input power of $15 \mathrm{dBm}$ because of the high effective resistivity compared to TR because of the effective resistivity value above $20 \mathrm{k} \Omega \mathrm{cm}$.

However, it is worth to know that Au-Si substrate has a different behavior at other DC bias points. The harmonics $\mathrm{H} 2$ and $\mathrm{H} 3$ measured at the output of the CPW line are plotted versus fundamental output power H1 in Fig. 9 for the $\mathrm{Au}-\mathrm{Si}$ substrate at different DC bias levels. The harmonic distortion is quite low at $0 \mathrm{~V}$ and comparable to TR (Fig. 11), however, for other DC bias points we register second harmonic distortion that are relatively high but still better than HR substrate.

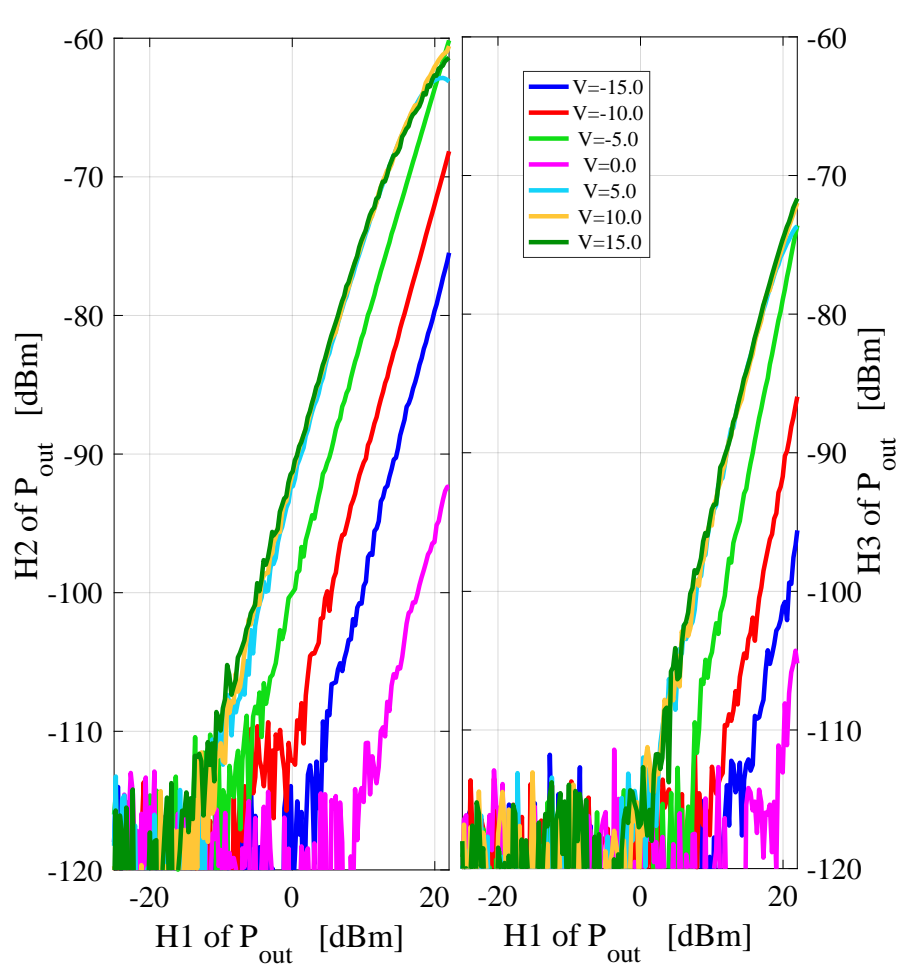

Fig. 9: Measured H2 (left) and H3 (right) for CPW line built on $\mathrm{Au}-\mathrm{Si}$ substrate versus fundamental $\mathrm{H} 1$ output power at $900 \mathrm{MHz}$.

\section{Non Linear Behavior Of AU-Si Substrate}

\section{A. Traps Distribution in the Silicon Band-Gap}

As mentioned above, the Au-Si substrate has many traps introduced in the silicon bandgap. After the introduction of the gold atoms in the silicon crystal, they occupy both substitutional and interstitial sites, the substitutional ones will take the $\mathrm{Si}$ atoms position thanks to the kick out mechanism [24], the interstitial ones will occupy a position within the crystall network. It was mentioned in different works that the gold atoms in substitutional $\left(\mathrm{Au}_{\mathrm{s}}\right)$ sites creates two energy levels of traps in the silicon bandgap, donor level at $\mathrm{E}_{\mathrm{c}}-0.78$ $\mathrm{eV}(0.78 \mathrm{eV}$ below the Conduction band) and acceptor level at $\mathrm{E}_{\mathrm{v}}+0.58 \mathrm{eV}(0.58 \mathrm{eV}$ above the valence band), however it was also mentioned in [25] that gold gold atoms in interstitial $\left(\mathrm{Au}_{\mathrm{I}}\right)$ sites creates donor traps at $\mathrm{E}_{\mathrm{v}}+0.43 \mathrm{eV}$. It was explained also that silicon atoms in inerstitial $\left(\mathrm{Si}_{\mathrm{I}}\right)$ sites creates acceptor traps at energy level $E_{v}+0.30 \mathrm{eV}$. Fig. 10 shows the distribution of the different traps in the silicon bandgap where we can see that the traps introduced are near the silicon midgap. However, the $\mathrm{Au}_{\mathrm{I}}$ and $\mathrm{Si}_{\mathrm{I}}$ will not participate to the increase of the resistivity of the substrate. 


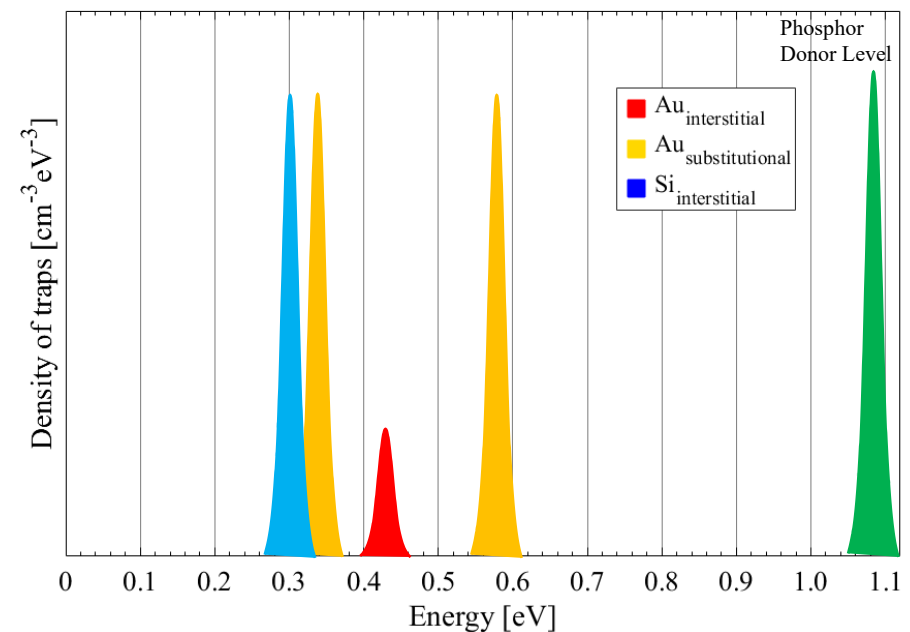

Fig. 10: Levels introduced by gold atoms (interstitial and substitutional sites) in the silicon bandgap.

\section{B. Fermi Level Localization}

The variation of the second harmonic $(\mathrm{H} 2)$ at an output fundamental power $\mathrm{H} 1=15 \mathrm{dBm}$ with the applied DC bias for the described substrates is depicted in Fig. 11.

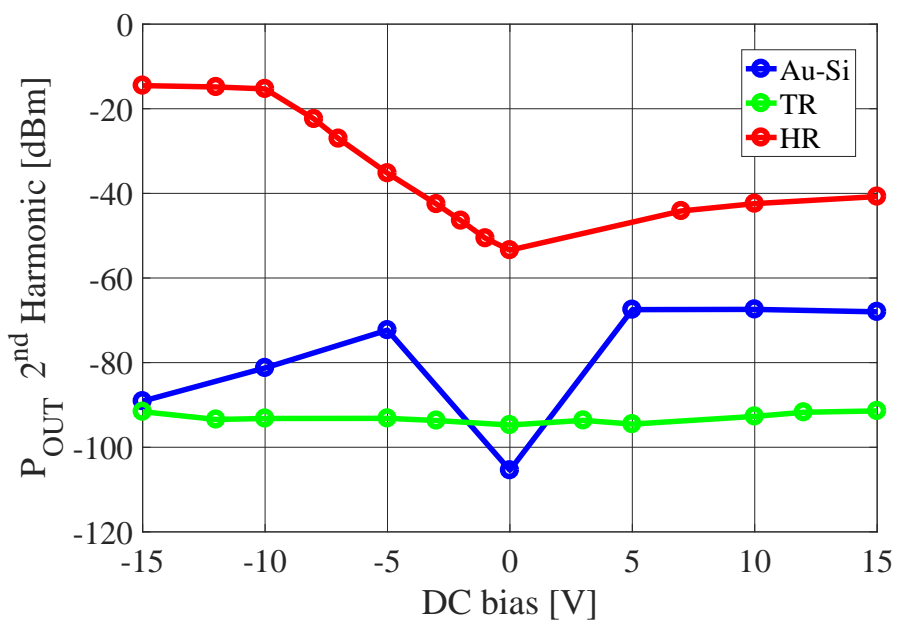

Fig. 11: Measured variation of the second harmonic at $\mathrm{H} 1=15$ $\mathrm{dBm}$ with the applied DC bias for the three different substrates.

While TR is almost bias independent (variation in a small range between -87 and $-90 \mathrm{dBm}$ ), the $\mathrm{HR}$ shows high $\mathrm{H} 2$ values and is strongly bias dependent varying from -14 to $-54 \mathrm{dBm}$ over the measured $\mathrm{DC}$ bias range. For the $\mathrm{Au}-\mathrm{Si}$ substrate the harmonic distortion (HD) is quite low at $0 \mathrm{~V}$ $(-105 \mathrm{dBm})$, however large degradations in $\mathrm{H} 2$ are observed at the other DC bias points (variation between -65 and $-90 \mathrm{dBm}$ ).

In order to explain the behavior of Au-Si under large signal, we simulated the Fermi level below the signal electrode of the $\mathrm{CPW}$ line just at the $\mathrm{Si}-\mathrm{SiO}_{2}$ interface and was represented in Fig. 12 as a function of the applied DC bias. Equation. 2 that shown the space charge density extracted from Poisson's equation:

$$
\rho=\mathrm{q}\left(\mathrm{p}-\mathrm{n}+\mathrm{N}_{\mathrm{D}}-\mathrm{N}_{\mathrm{A}}+\rho_{\mathrm{p}}-\rho_{\mathrm{n}}\right)
$$

where $q$ is the elementary charge, $p$ and $n$ the hole and electron concentrations, respectively. $N_{\mathrm{D}}, N_{\mathrm{A}}, \rho_{p}$ and $\rho_{n}$ are donor impurities, acceptor impurities, trapped holes and electrons, respectively.

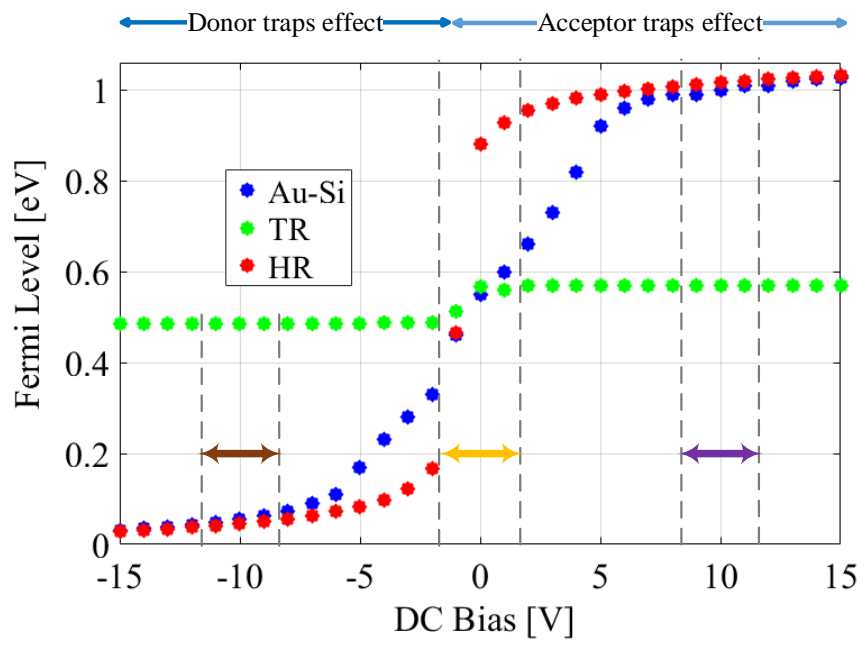

Fig. 12: Variation of the Fermi level as a function of the DC bias points for Si-based substrates.

It is well known that the HR substrate does not contain traps in the silicon bandgap $\left(\rho_{p}-\rho_{n}=0\right)$, so the applied DC bias is compensated by a large variation of in free carriers consequently a large variation in the Fermi level as shown in Fig. 12. For the TR substrate the Fermi level is pinned near the midgap thanks to the polysilicon layer that introduces high trap density, so the applied DC bias is compensated by activating and deactivating the $\operatorname{acceptor}\left(\rho_{n}\right)$ and $\operatorname{donor}\left(\rho_{p}\right)$ traps, the value of $\rho$ in the Equation. 2 will remain almost constant whatever the value of the applied DC value. The AuSi substrate shows a different Fermi level behavior comparing to $\mathrm{HR}$ and TR. Around $0 \mathrm{~V}$ DC bias, when a large signal $(15 \mathrm{dBm})$ is applied (a sine wave of $2 \times 1.7 \mathrm{~V}$ pic to pic), the Fermi level will oscillate between $\mathrm{E}_{\mathrm{v}}+0.62 \mathrm{eV}$ and $\mathrm{E}_{\mathrm{v}}+0.4 \mathrm{eV}$ (yellow arrow), the traps are able to compensate this DC bias variation by activating and deactivation donor and acceptor traps. In contrast, at $10 \mathrm{~V}$ DC bias, when the same large signal is applied, a high density of electrons is generated in the substrate the Fermi level will oscillate near the conduction band between $\mathrm{E}_{\mathrm{v}}+0.98 \mathrm{eV}$ and $\mathrm{E}_{\mathrm{v}}+1.01 \mathrm{eV}$ (purple arrow), this is because the traps density is not large enough to compensate the applied DC bias. the same for $-10 \mathrm{~V}$ DC bias, where we will have a high density of holes and the traps can not compensate this amount, consequently the Fermi level oscillate between $\mathrm{E}_{\mathrm{v}}+0.01 \mathrm{eV}$ and $\mathrm{E}_{\mathrm{v}}+0.06 \mathrm{eV}$ (brown arrow).

The position of the Fermi level near the mid-gap allow us to say that the linearity of the substrate is high enough, however when its position is close to one of the bands we will have an excess of free carriers that will be modulated by the applied signal thus lower linearity, this is why we found a low harmonic distortion for $0 \mathrm{~V}$ and higher harmonic for other DC bias. 
It is worth to know that the acceptor traps are activated only when a positive bias is applied because the electrons are generated by a positive DC bias, however the donor ones will be activated only for the negative DC bias due to the holes generated by this latter in the substrate.

\section{Impedance Seen by the CPW Line}

The non-linearities of the studied substrates are described using impedances network at the $\mathrm{Si}-\mathrm{SiO}_{2}$ interface. As shown in Fig. 13, $\mathrm{Z}_{\text {signal }}$ presents the impedance below the signal electrode and $\mathrm{Z}_{\text {sig-gnd }}$ is the impedance at the $\mathrm{Si}_{-} \mathrm{SiO}_{2}$ interface between signal and ground electrodes.

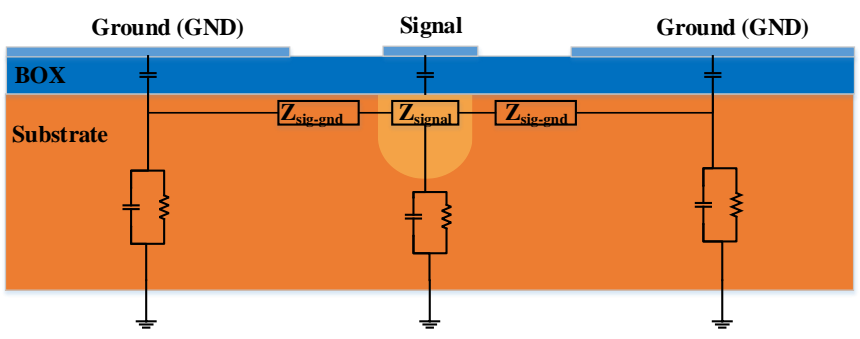

Fig. 13: Substrate modeling using the impedance seen by the coplanar waveguide line (CPW).

The HR substrate presents a highly non linear impedance $\mathrm{Z}_{\text {signal }}$ (very low impedance) that is in series with very low impedance $\mathrm{Z}_{\text {sig-gnd }}$ due to the large quantity of electrons (PSC effect), thus the overall impedance is low, so the substrate is non-linear. In contrast to $\mathrm{HR}$, the $\mathrm{TR}$ substrate shows a high $\mathrm{Z}_{\text {sig-gnd }}$ impedance as it overcomes the PSC effect, and relatively low variation below the signal electrode $\left(Z_{\text {signal }}\right)$ as shown in Fig. 12, so the overall impedance seen by the CPW line is high and can not be influenced by the large signal applied to it. This explains the high linearity of the TR substrate and its bias independance.

The non-linear behavior of the substrate beneath the central CPW line on the Au-Si substrate is similar to that on HR, as Fig. 12 shows. The interface Fermi level is not pinned by the traps as in the TR case, so we have a highly nonlinear impedance $\mathrm{Z}_{\text {signal }}$. However, in the Au-Si substrate this non-linear impedance $\left(Z_{\text {signal }}\right)$ comes in series with a highly resistive impedance $Z_{\text {sig-gnd }}$, and so its contribution to the overall CPW shunt impedance is attenuated, and as a result the harmonic distortion is significantly lower than for HR. Table. I summerizes the linearity (variations) of $\mathrm{Z}_{\text {signal }}$ and the value of the series impedance $Z_{\text {sig-gnd }}$ (the overall impedance linearity is the sum of the two latters) of the CPW line lying on the Si-based substrates.

\section{Effect of Traps Concentration}

Fig. 14 shows the simulated Fermi level for Au-Si substrate as a function of the applied DC bias with different gold atoms densities, and this starting from standard substrate $(56 \Omega-\mathrm{cm})$
TABLE I: Linearity comparison between the studied substrates.

\begin{tabular}{cccc}
\hline & $Z_{\text {signal }}$ & $Z_{\text {signal-ground }}$ & Linearity \\
\hline \hline $\mathrm{HR}$ & Strong variation & Low & Poor \\
$\mathrm{Au}-\mathrm{Si}$ & Strong variation & High & Medium \\
$\mathrm{TR}$ & Little variation & High & High \\
\hline
\end{tabular}

until reaching a concentration of $1 \times 10^{17} \mathrm{~cm}^{-3}$. These densities are introduced uniformly in the entier thickness $(700 \mu \mathrm{m}$ of the substrate. It is clear to notice that the highly doped substrate (std) presents Fermi level that oscillates between the two bands (with only one point near the mid-gap at $\mathrm{DC}=-1 \mathrm{~V}$ ), in order to compensate the free carriers generated by the DC bias, however by increasing the density of traps, we notice that the sweeping domain of the Fermi level is shrinking more and more and the Fermi level at $0 \mathrm{~V}$ is going close to the midgap. It would be great to go for densities more than $1 \times 10^{17}$ $\mathrm{cm}^{-3}$, however the solubility of gold in silicon is limited to this value. We can simulate for quantities more than that the value described before, but it does not have a physical meaning.

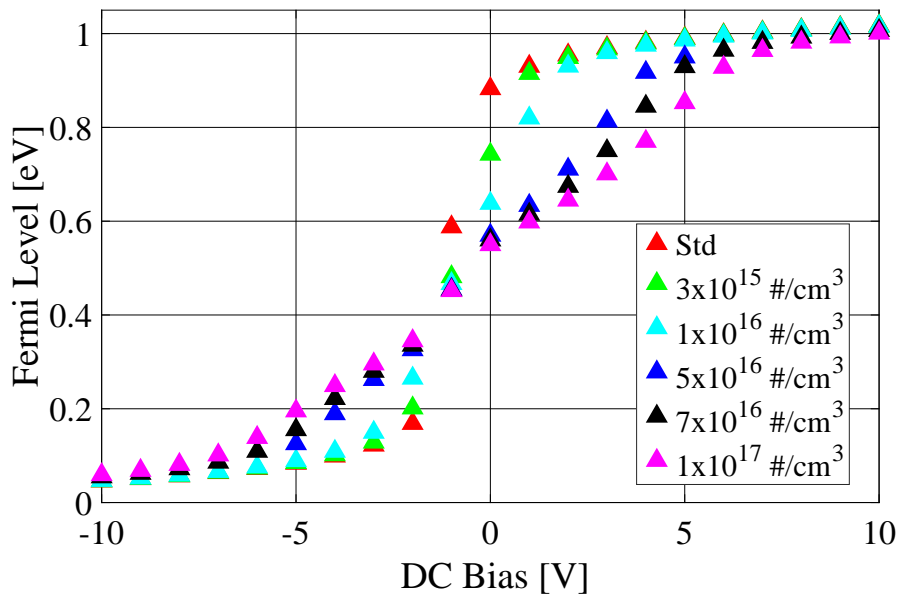

Fig. 14: Simulated Fermi level for Au-Si substrate with different densities of gold atoms

\section{CONCLUSION}

In this paper we, demonstrate that we cannot rely on the unique extraction of the effective resistivity of a substrate to predict its harmonic distortion, we saw that the $\mathrm{Au}-\mathrm{Si}$ show similar small signal performances such as crosstalk, quality factor of the inductors and the shape of the band pass filter. The harmonic distortion in $\mathrm{Au}-\mathrm{Si}$ is lower than in HR because though the Fermi level and local resistivity beneath the signal line of the CPW varies strongly in both substrates in response to the large signal, the $\mathrm{Si}_{-} \mathrm{SiO}_{2}$ interface is in a highly conductive state (PSC) for the HR, but is highly resistive for the Au-Si. Indeed, the gold traps are in sufficient quantity to effectively counter the oxide charges, and add a high substrate impedance in series with the voltage sensitive region below the signal line that enables lower HD and higher effective resistivity than HR. The traps are however not sufficient to fully pin the Fermi-level such as is the case in 
the TR sample, which therefore presents much lower harmonic distortion. Eventhough the Au-Si substrate is not suited is the industry due to its high diffusion and show a considerable variations in the HD, it still have a low level HD and we can say that it could be one of the contenders of the TR in the microelectronic market.

\section{REFERENCES}

[1] S. Laurent and D. Eric, "RF Substrate Technologies for mobile communications," white paper, may 2011.

[2] M. Rack, Y. Belaroussi, K. B. Ali, G. Scheen, B. K. Esfeh, and J.-P. Raskin, "Small-and Large-Signal Performance Up To 175 C of LowCost Porous Silicon Substrate for RF Applications," IEEE Transactions on Electron Devices, vol. 65, no. 5, pp. 1887-1895, 2018.

[3] K. B. Ali, C. R. Neve, A. Gharsallah, and J.-P. Raskin, "RF performance of SOI CMOS technology on commercial 200-mm enhanced signal integrity high resistivity SOI substrate," IEEE Transactions on Electron Devices, vol. 61, no. 3, pp. 722-728, 2014.

[4] D. Lederer and J.-P. Raskin, "Effective resistivity of fully-processed SOI substrates," Solid-State Electronics, vol. 49, no. 3, pp. 491-496, 2005.

[5] K. B. Ali, C. R. Neve, Y. Shim, M. Rais-Zadeh, and J.-P. Raskin, "Nonlinear characteristics of passive elements on trap-rich high-resistivity Si substrates," in Silicon Monolithic Integrated Circuits in RF Systems (SiRF), 2014 IEEE 14th Topical Meeting on. IEEE, 2014, pp. 4-6.

[6] G. Celler and S. Cristoloveanu, "Frontiers of silicon-on-insulator," Journal of Applied Physics, vol. 93, no. 9, pp. 4955-4978, 2003.

[7] A. Tombak, M. S. Carroll, D. C. Kerr, J.-B. Pierres, and E. Spears, "Design of high-order switches for multimode applications on a siliconon-insulator technology," IEEE Transactions on Microwave Theory and Techniques, vol. 61, no. 10, pp. 3639-3649, 2013

[8] A. O. Adan, T. Yoshimasu, S. Shitara, N. Tanba, and M. Fukurni, "Linearity and low-noise performance of SOI MOSFETs for RF applications," IEEE transactions on Electron Devices, vol. 49, no. 5, pp. 881-888, 2002.

[9] D. Lederer and J.-P. Raskin, "RF performance of a commercial SOI technology transferred onto a passivated HR silicon substrate," IEEE Transactions on Electron Devices, vol. 55, no. 7, pp. 1664-1671, 2008.

[10] F. Gianesello, D. Gloria, C. Raynaud, S. Montusclat, S. Boret, and P. Touret, "Integrated inductors in HR SOI CMOS technologies: on the economic advantage of SOI technologies for the integration of RF applications," in SOI Conference, 2007 IEEE International. IEEE, 2007, pp. $119-120$.

[11] N. Z. Hashim, A. Abuelgasim, and C. H. de Groot, "Suppression of parasitic surface conduction in Au-compensated high resistivity silicon for 40-GHz RF-MMIC application," in Microwave Conference (APMC), 2014 Asia-Pacific. IEEE, 2014, pp. 55-57.

[12] A. Abuelgasim, "High resistivity Czochralski-silicon using deep level dopant compensation for RF passive devices," Ph.D. dissertation, University of Southampton, 2012.

[13] C. Collins, R. Carlson, and C. Gallagher, "Properties of gold-doped silicon," Physical Review, vol. 105, no. 4, p. 1168, 1957.

[14] W. Bullis, "Properties of gold in silicon," Solid-State Electronics, vol. 9, no. 2, pp. 143-168, 1966 .

[15] M. Morooka, H. Tomokage, H. Kitagawa, and M. Yoshida, "Three states of substitutional gold in silicon," Japanese Journal of Applied Physics, vol. 24, no. 2R, p. 133, 1985.

[16] G. Bemski, "Recombination properties of gold in silicon," Physical Review, vol. 111 , no. 6, p. 1515,1958

[17] A. Abuelgasim, K. Mallik, P. Ashburn, D. Jordan, P. Wilshaw, R. Falster, and C. de Groot, "Reduced microwave attenuation in coplanar waveguides using deep level impurity compensated Czochralski-silicon substrates," Semiconductor Science and Technology, vol. 26, no. 7, p. 072001, 2011.

[18] A. Abuelgasim, N. Hashim, H. Chong, P. Ashburn, and C. De Groot, "Low loss $67-\mathrm{GHz}$ coplanar waveguides and spiral inductors on 100 $\mathrm{k} \Omega \mathrm{cm}$ gold-doped high resistivity Cz-Silicon," in Silicon Monolithic Integrated Circuits in Rf Systems (SiRF), 2014 IEEE 14th Topical Meeting on. IEEE, 2014, pp. 10-12.

[19] D. S. Atlas, "Atlas users manual," Silvaco International Software, Santa Clara, CA, USA, 2005

[20] M. Kemp, M. Meunier, and C. Tannous, "Simulation of the amorphous silicon static induction transistor," Solid-state electronics, vol. 32, no. 2, pp. 149-157, 1989.
[21] R. Gillon, J.-P. Raskin, D. Vanhoenacker, and J.-P. Colinge, "Determining the reference impedance of on-wafer TLR calibrations on tossy substrates," in 1996 26th European Microwave Conference, vol. 1. IEEE, 1996, pp. 170-173.

[22] R. Hull, Properties of crystalline silicon. IET, 1999, no. 20.

[23] C. R. Neve and J.-P. Raskin, "RF harmonic distortion of CPW lines on HR-Si and trap-rich HR-Si substrates," IEEE Transactions on Electron Devices, vol. 59, no. 4, pp. 924-932, 2012.

[24] U. Gösele, F. Morehead, W. Frank, and A. Seeger, "Diffusion of gold in silicon: A new model," Applied Physics Letters, vol. 38, no. 3, pp. 157-159, 1981.

[25] H. Bracht and A. Rodriguez Schachtrup, "Diffusion of Gold into Heavily Boron-Doped Silicon,” MRS Proceedings, vol. 469, p. 25, 1997.

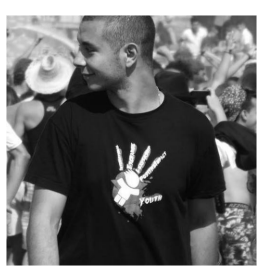

Massinissa Nabet received the M.S. degree in electronic systems instrumentation from the Université des Sciences et de la Technologie Houari Boumediene, Algeria, in 2016, where he is currently pursuing the Ph.D. degree in collaboration with UCLouvain.

His current research interests include modelling, synthesis and high-frequency characterization of RF CMOS devices and semiconductor substrates for the high-frequency communications.

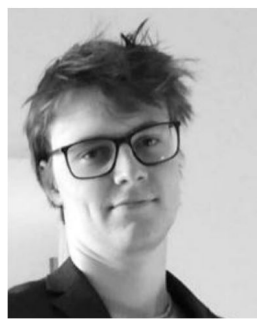

Martin Rack received the M.S. degree in electrical engineering from the Université catholique de Louvain, Belgium, in 2014, where he is currently pursuing the $\mathrm{Ph} . \mathrm{D}$. degree.

His current research interests include small and large-signal modeling of RF CMOS devices and semiconductor substrates, and their characterization and optimization toward successful monolithic integration of RF devices using silicon based technology.

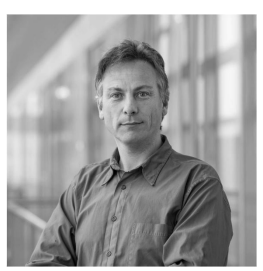

C. H. (Kees) de Groot received a Masters degree in Physics in 1994 from the University of Groningen, the Netherlands. His main interest is the integration of novel nano-materials and devices with silicon electronics processing with particular emphasis on the semiconducting and dielectric properties of oxides, chalcogenides, and carbides. His recent breakthroughs in these areas includes the first $100 \mathrm{~nm} \mathrm{GeSbTe}$ phase change memory by non-aqueous electrodeposition, and functional oxide nanostructures resulting in plasmonic devices with ultrafast modulation of optical and dielectric properties of ITO, AZO and VO2 by optical, electrical, and thermal methods.

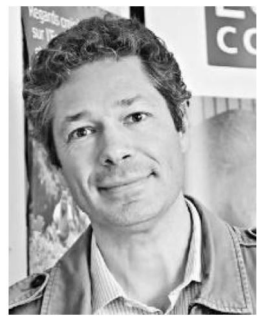

Jean-Pierre Raskin received the M.S. and Ph.D. degrees in applied sciences from the Université catholique de Louvain, Louvain-la-Neuve, Belgium, in 1994 and 1997, respectively. $\mathrm{He}$ has been a Professor with the Universite catholique de Louvain since 2000. His current research interests include the modeling, wideband characterization and fabrication of advanced SOI MOSFETs, and substrates toward RF integration of SOI technology. 